\title{
The Use of an Inertial Motion Analysis System to Evaluate the Kinematics of Landing before and after a Six-Session Training Intervention Focused on Proximal Hip Strengthening, Motor Control, and Lower Extremity Loading Strategies
}

\author{
Kevin Robinson*, Craig Parker, Emily Jones, Jenny Ellison, Abby Lester, Kayla Lydon, \\ Megan Rolfe, Pat Sells
}

Belmont University, School of Physical Therapy, Nashville, TN, USA

Email: ^kevin.robinson@belmont.edu

How to cite this paper: Robinson, K., Parker, C., Jones, E., Ellison, J., Lester, A., Lydon, K., Rolfe, M. and Sells, P. (2019) The Use of an Inertial Motion Analysis System to Evaluate the Kinematics of Landing before and after a Six-Session Training Intervention Focused on Proximal Hip Strengthening, Motor Control, and Lower Extremity Loading Strategies. Open Access Library Journal, 6: e5898. https://doi.org/10.4236/oalib.1105898

Received: November 2, 2019

Accepted: November 24, 2019

Published: November 27, 2019

Copyright () 2019 by author(s) and Open Access Library Inc.

This work is licensed under the Creative Commons Attribution International License (CC BY 4.0).

http://creativecommons.org/licenses/by/4.0/

\begin{abstract}
Background/Purpose: Studies investigating the trends of anterior cruciate ligament (ACL) reconstruction rates have shown that since 2006, females aged $13-17$ years of age have had the highest injury rates of any age or gender group in the country. This study investigated the lower chain biomechanics of landing before and after a six-session training intervention. This training session was conducted between the fall and spring season break for a travel soccer club. Study design: Non-randomized controlled follow-up study. Methods: Thirty-six healthy, female soccer players between the ages of ten and fifteen years old participated. Using an inertial tracking system for three-dimensional kinematic (motion) analyses of a drop jump assessment that were conducted before and after a six-session training intervention focused on proximal hip strengthening, motor control, and lower extremity loading strategies. The study took place over a period of eight weeks. Results: When compared to pre-intervention measures for the drop jump, post-intervention measures were significantly improved bilaterally for each of the following variables: knee flexion ( $p=0.000)$, hip flexion ( $p=0.000)$, and hip abduction $(\mathrm{p}=0.002$. As compared to pre-test jump height, post-test jump heights were significantly lower by an average value of $1.8034 \mathrm{~cm}(\mathrm{p}=0.005)$. Conclusions: The results of this study showed that a short duration (six, $90 \mathrm{~min}$, sessions occurring over 8 weeks) training intervention can significantly influence the landing
\end{abstract}


kinematics in adolescent female soccer players during a drop jump assessment. Increased peak values of hip and knee flexion along with decreased hip adduction during landing indicate a more optimal loading technique associated with decreased risk of ACL injury. The inertial motion analysis system was a useful screening tool for identifying high-risk biomechanics that must be addressed to develop successful ACL injury prevention programs.

\section{Subject Areas}

Orthopedics

\section{Keywords}

ACL Injury Prevention, Landing Kinematics, Female Soccer Players

\section{Introduction}

Injuries to the anterior cruciate ligament occur at a high rate in the athletic population with an estimated 0.7 - 2.5 ACL tears per every 1000 athletic exposures [1]. Of all sports, ACL injuries are especially common in soccer, the most widely played sport in the world, among both males and females [2]. The consequences of injury can be detrimental to the athlete physically, mentally, and financially, while placing a burden on the nation's public health system as well.

The financial toll of an ACL injury is substantial in that the approximate cost to society for ACL reconstruction is estimated to be $\$ 38,121$, while the cost of rehabilitation without surgery is approximately $\$ 88,538$ [3]. Furthermore, ACL injury puts a person at high risk for osteoarthritis later in life, making injury a lifetime burden on both the individual and the economy. As many as $13.9 \%$ of young adults with a history of knee injury will have knee osteoarthritis by the age of 65 , as compared to $6 \%$ of young adults without a history of knee injury [4]. An ACL injury accompanied by meniscal injury can lead to knee osteoarthritis in $21 \%$ - $48 \%$ of individuals [5]. In female soccer players examined 12 years after ACL injury, $82 \%$ showed osteophyte formation and joint space narrowing of the knee on radiographs [6].

ACL injury also takes time away from the athlete's participation in sport. An analysis conducted among patients from the Multicenter Orthopaedic Outcomes Network (MOON) investigated return to play (RTP) among soccer players after ACL reconstruction. Of 100 subjects, $76 \%$ of males and $67 \%$ of females returned to soccer after ACL reconstruction. However, the average RTP time was $12.2 \pm$ 14.3 months, indicating that ACL injury results in loss of an entire season for each athlete injured. The results also demonstrated that RTP is slightly less in females than in males [7].

Injury to the ACL can occur by contact or non-contact mechanisms. Previous literature has shown that approximately $70 \%$ of $\mathrm{ACL}$ injuries occur in non-contact 
situations (without bodily contact from another player or object) [8]. Of non-contact injuries, most tears occur during rapid changes in direction associated with deceleration, particularly when there is an increase in knee extension torque and/or dynamic valgus moment at the knee when the ipsilateral foot is planted on the ground. This can occur when landing from jumping with the knee at or near full extension, during rapid change of direction moments in dynamic valgus positioning, or when pivoting near extension on a planted foot [9] [10] [11]. The risk of injury is further increased when any of the above occurs in combination with a strong quadriceps contraction. At full knee extension, the angle-of-insertion of the patellar tendon is greatest relative to the tibia, making the ACL vulnerable and increasing the risk for a tear [12]. Fatigue has also been shown to decrease knee flexion and increase hip internal rotation during dynamic closed-chain deceleration moments, further increasing the risk for injury [13].

Risk factors for ACL injury are both modifiable and non-modifiable in nature. Non-modifiable factors can be environmental or biological. Environmental factors include playing surface, weather, bracing, and footwear [14], while biological factors include limb alignment, joint instability, ligament size [15], hormones, and genetic predisposition to any of the aforementioned biological aspects. While distinct and important for consideration, such anatomical, hormonal, and genetic risk factors are not modifiable in current medical practice. Thus, intervention strategies to decrease injury risk are consequently directed towards those that are modifiable in nature. Specifically, targeting risk factors associated with neuromuscular control and biomechanics which are susceptible to change with training interventions has shown the greatest potential to reduce the incidence of non-contact ACL injury when addressed effectively [16] [17] [18] [19].

Females in general are known to be at higher risk for ACL injury, in part due to the greater influence of anatomical and hormonal risk factors in this population relative to males. In a study of private insurance data for 148 million U.S. residents found that overall, the average annual anterior cruciate ligament (ACL) surgery rate climbed 22 percent from 2002 to 2014, when it reached 75 procedures for every 100,000 people. However for teen girls, the average annual knee surgery rate rose by 59 percent during the study period to 269 procedures for every 100,000 people [20]. Female athletes who play soccer and basketball are two-to-eight times more likely to suffer an ACL injury compared to their male counterparts, according to the American Orthopaedic Society for Sports Medicine [21]. Further, females tend to exhibit altered neuromuscular strategies during the stance phase of lower extremity loading maneuvers when compared to their male counterparts. Such neuromuscular strategies alter the distribution of forces through the knee, making them disadvantageous. Strategies implemented by females tend to include less knee flexion angles, greater knee valgus angles, greater quadriceps activation, and lower gluteal and hamstring activation in comparison to strategies implemented by males [22]. Females have also been shown to exhibit greater tibial internal rotation than males, which further increases the risk of ACL injury [23] [24]. 
A 10-year study investigating male and female NCAA basketball and soccer players found that females are three times more likely than males to sustain an ACL tear through non-contact mechanisms. When in contact with another player, females are twice as likely as men to injure their ACL. In particular, females are more likely to exhibit a valgus moment at the knee during deceleration, while males typically experience a varus moment [25]. A large valgus moment puts a greater strain on the ACL when compared to non-valgus loading when the knee is flexed in closed-chain movement [26]. Peak frontal plane movements have been shown to be twice as high in females than in males, and the ACL is one of the primary passive stabilizing structures when the knee is loaded in the frontal plane of motion [25]. Plyometric training has been shown to decrease both knee valgus moments and peak impact forces, and therefore may be warranted for utilization in preventative training interventions [19].

Along with a tendency to over-activate the quadriceps, females also demonstrate suboptimal neuromuscular control during skilled performance involving complex dynamic loading actions of the lower quarter [24]. Neuromuscular insufficiency of this nature predisposes athletes to injury and specifically can lead to ACL strain or rupture when the knee is loaded in known positions of high-risk [9]. Women are more likely than men to exhibit movement behavior that places high stress on the ACL during closed chain loading moments of the lower quarter, although there is debate in the field regarding which factors contribute the most to this tendency [22] [27]. It has been proposed that the coordination of muscular contractions directly influences the loading of the ACL. Co-contraction of the quadriceps and hamstrings at the knee joint causes joint compression, which may protect the joint from anterior translational force [22] [28]. Thus, joint compression may allow for increased valgus loading at articular contact forces, which could decrease the load to the ligaments, protecting their integrity.

When comparing male and female kinetics during side-step cutting, it has been shown that females exhibit greater quadriceps activation on EMG than males. Along with increased frontal plane movement, females also demonstrate a smaller peak knee flexor moment and higher adductor moment, which also may reduce sagittal plane protection and allow for larger anterior tibial translation [25]. The tendency to over-activate the quadriceps during landing is known as a "stiff landing" (low flexion) technique, which results in limited hip and knee flexion. Females tend to exhibit a "stiff landing," while males typically demonstrate a "soft landing" (high flexion) technique that involves greater hamstring activation. The "stiff landing" technique has been shown to cause increased frontal plane loading during deceleration, as well as decreased energy absorption at the hip. It has also been demonstrated that females exhibit less knee flexion than males during running, side-cutting, and cross-cutting functional activities [22] [23].

Neuromuscular insufficiency and suboptimal regional coordination patterns are elements that can be addressed by an ACL injury prevention program. Such interventions are designed to improve modifiable risk factors related to ACL trauma, such as muscular strength, technique, and body mechanics during landing 
and cutting movements.

The purpose of this study was to utilize an inertial tracking system to investigate the biomechanics of jumping and landing before and after a six-session training intervention focused on proximal hip strengthening, motor control, and lower extremity loading strategies. The mean values of the hip, knee, and ankle joints were analyzed in a population of healthy, young, female soccer players. Secondary aims were to determine whether jump height increased, decreased, or remained the same following intervention.

\section{Methods}

\subsection{Subjects}

A total of 54 female athletes between the ages of 10 and 15 years old were recruited from a local soccer club for a six-session training intervention, which was completed over the course of eight weeks. The exclusion criteria used was an athlete who currently had a lower extremity injury or had undergone a surgery on their lower extremity within the past two years. Overall, 36 of the participating athletes agreed to undergo clinical testing and motion analysis assessments before and after the training intervention.

\subsection{Instrumentation}

Noraxon's Myo Motion inertial system is a three-dimensional kinematic system that measures joint position using compact Inertial Measurement Units (IMUs). Measurements are obtained from sensors placed on specific segments of the body, which determine the three-dimensional angular orientation of the body parts they overlie. The placement of multiple sensors allows for analysis of joint articulation of the entire body. The inertial system used in this study was manufactured by Noraxon. It records measurements with a static accuracy of \pm 0.4 degrees and a dynamic accuracy of \pm 1.2 degrees [29] with three degrees of freedom.

The Vertec Vertical Jump is an open design that allows vertical jump measurements accurate to the nearest 0.5 inch. Vertec is manufactured by Jump USA. The equipment includes a reset pole that allows the testing administrator to record jump heights from 6 - 12 feet. In this study, measurements were converted to centimeters for recording purposes.

\subsection{Procedure}

Prior to data collection, the study was approved by Belmont University's Institutional Review Board in. Each athlete and guardian received a child assent form and parental informed consent to review and sign. The athlete was then assigned an identification number. Participants completed the motion analysis activities individually and in series while being assessed by a team of physical therapists (PT) and physical therapy students (SPT). Each SPT was under direct supervision of the present PTs.

Biometric data of height and weight, along with previous history of injury, was recorded for each subject. A brief lower quarter screen was then performed 
on each participant to identify any potential red flags not otherwise reported. Screening included the following: performance of a full bilateral squat, hip scouring test, Femoral-Acetabular Impingement (FAI) test, Flexion Abduction External Rotation (FABER) test, and bilateral resisted manual muscle testing of hip flexors, knee extensors, knee flexors, and ankle dorsiflexors. Participants who reported any previous injuries in the lower quarter were screened appropriately for ligamentous integrity in the respective regions of involvement. Subjects with injury present at the time of the study were excluded from testing.

\subsection{Motion Analysis}

MyoMotion sensors were placed on each participant at the following locations: over the sacrum, mid-thighs (bilaterally), midway foreleg (bilaterally), and dorsum of the feet (bilaterally). Participants were instructed to complete a Drop Jump Test from a $40 \mathrm{~cm}$ box. Image 1(a) shows the placement of MyoMotion sensors and the starting position for the test. Each participant started atop the box and was instructed to jump down from the box to the floor. Upon landing, the participants were told to jump vertically as high as possible and with maximum effort for three different trials. During each jump, MyoMotion sensors recorded biomechanical data of hip, knee, and ankle joint movements. Simultaneously, vertical jump height measurements were obtained in inches using the Vertec Vertical Jump. Image 1(b) shows a progression of the jump from take-off, landing, and peak vertical jump height in the sagittal view. Image 1(c) depicts peak jump height and alignment of limbs in the frontal view.

In order to control for the variation of height among subjects, each participant stood on tiptoes under the Vertec and reached up to touch as many Vertec units as possible. These units were then moved aside and used as a landmark from which Drop Jump measurements were taken. Participants stood on the $40 \mathrm{~cm}$ box with feet shoulder-width apart to calibrate the MyoMotion. Each subject was

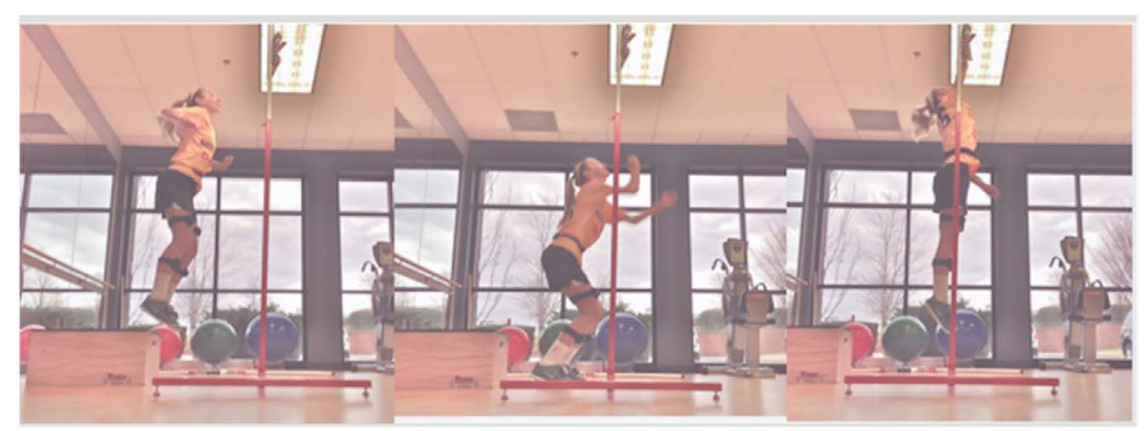

(a)

(b)

(c)

Image 1. Sequence of drop jump followed by vertical jump. (a) Shows the placement of MyoMotion sensors and the starting position for the test. Each participant started atop the box and was instructed to jump down from the box to the floor; (b) Upon landing, the participants were told to jump vertically as high as possible and with maximum effort for three different trials. During each jump, MyoMotion sensors recorded biomechanical data of all the joints of the lower extremities; (c) Depicts peak vertical jump and simultaneously, vertical jump height measurements were obtained in inches using the Vertec. 
then instructed to jump from the $40 \mathrm{~cm}$ box to the floor and to jump upwards immediately on landing. While jumping upwards, participants touched as many Vertec units as possible by reaching with their upper extremity. Data collected from the MyoMotion sensors was recorded after each jump. After the participant completed three jump tests, the Vertec was recalibrated for the next participant.

Following pre-testing procedures, a six-session neuromuscular training intervention was implemented over the course of eight weeks. Participants were divided into two groups of younger and older divisions respectively, and trained by a licensed physical therapist for six, 90 minute sessions spaced over the duration of the study. Training methods primarily emphasized proximal hip strengthening, motor control in various unilateral weight bearing positions, and general lower extremity loading strategies specific to dynamic pivoting sports including jumping, landing, and rapid change of direction moments. Following the training intervention, pre-testing procedures were repeated for post-test data collection and analysis.

\section{Results}

Thirty-six total females participated in pre-testing, intervention, and post-test measures. The group as a whole attended approximately 5.5 of 6 sessions on average. All individuals were free of injury, and no complaints of pain were reported during testing. Prior to statistical analysis, data were evaluated to ensure all parametric assumptions were met for each of the variables investigated. Data were analyzed with IBM SPSS Statistics, software version 22.0. Statistical significance was quantified as a p-value $\leq 0.05$.

\subsection{Drop Jump Test, Knee Flexion}

A mixed two-way ANOVA $(2 \times 2)$ indicated a significant difference $(\mathrm{p}=0.000)$ in the mean peak knee flexion angle in the Drop Jump Test pre-test vs. post-test in the MyoMotion assessment. The mean peak knee flexion angle was significantly higher in the post-test $\left(99.323^{\circ}\right)$ assessment when compared to pre-test $\left(90.718^{\circ}\right)$, as illustrated in Figure 1.



Figure 1. Sagittal plane knee motion. Significant differences $(p=0.000)$ were seen in the post training data for both knees. 
When testing the second null hypothesis examining the main effect of side of the body (left versus right), a significant difference $(\mathrm{p}=0.041$ ) was indicated with the mixed two-way ANOVA. The results indicate that in the population tested, the left side of the body had a significantly greater degree of knee flexion than the right side. The ANOVA also indicated that there was no significant interaction ( $\mathrm{p}=0.058$ ) between time and side of body.

The mean score for right knee flexion increased from a score of $87.9231^{\circ}$ pre-training to $98.9682^{\circ}$ post-training. The mean score for left knee flexion increased from a score of $93.5120^{\circ}$ pre-training to $99.6787^{\circ}$ post-training. These results indicate that in the population tested, the right side of the body had a significantly higher score than the left side.

\subsection{Drop Jump Test, Hip Flexion}

A mixed two-way ANOVA $(2 \times 2)$ indicated a significant difference $(\mathrm{p}=0.000)$ in time comparing the mean peak hip flexion angle in the Drop Jump Test pre-test vs. post-test. The mean peak hip flexion angle was significantly higher in the post-test $\left(77.826^{\circ}\right)$ assessment when compared to pre-test $\left(67.145^{\circ}\right)$, as illustrated in Figure 2.

When testing the second null hypothesis examining the main effect of side of body (left versus right), no significant difference $(p=0.056$ ) was indicated with the mixed two-way ANOVA. These results indicate that in the population tested, the right side did not have significantly higher score than the left.

The ANOVA indicated a significant interaction between time and side of body. When considering the significant interaction associated with ANOVA ( $\mathrm{p}$ $=0.039$ ), further differences were illuminated. When comparing pre-training values of right and left hip flexion angles, the right side was significantly greater $(\mathrm{p}=0.001)$. There was no significant difference in post-training values between the left and right hip flexion angles $(p=0.754)$. All post-hocs were completed with a paired samples t-test and a Bonferroni correction with a critical p-value of 0.025 .

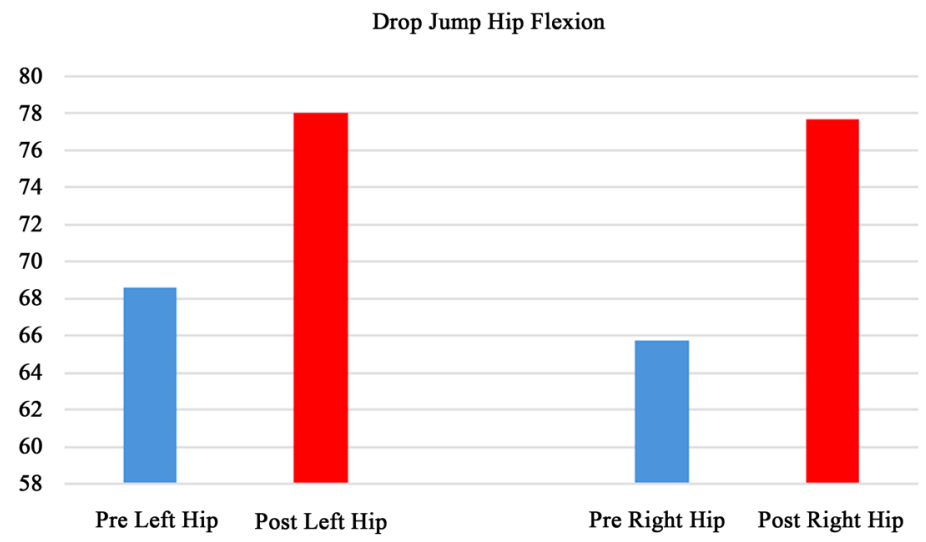

Figure 2. Sagittal plane hip motion. Significant differences $(p=0.000)$ were seen in the post training data for both hips. 
The mean value for right peak hip flexion increased from $65.719^{\circ}$ pre-training to $77.657^{\circ}$ post-training. The mean value for left peak hip flexion increased from a score of $68.571^{\circ}$ pre-training to $77.994^{\circ}$ post-training. These results indicate that participants increased their total peak flexion excursion a greater amount on the right side compared to the left.

\subsection{Drop Jump Test, Hip Abduction}

A mixed two-way ANOVA indicated a significant difference $(\mathrm{p}=0.002)$ in time, pre-test and post-test. The post-test hip abduction mean was significantly higher mean than the pre-test mean. The pre-test mean was $4.060^{\circ}$ of adduction and the post-test mean was $1.461^{\circ}$ of adduction, as illustrated in Figure 3.

When testing the second null hypothesis o examine the main effect of "side of body" (left versus right), a significant difference $(p=0.000)$ was indicated with the mixed two-way ANOVA. These results indicate that in the population tested, the right was in more adduction than the left.

The ANOVA indicated that there was no significant interaction $(\mathrm{p}=0.465)$ between time and side of body. The mean value for right hip abduction improved from a score of $8.456^{\circ}$ of adduction pre-training to $5.255^{\circ}$ of adduction post-training. The mean value for left hip abduction increased from a score of $0.335^{\circ}$ of abduction pre-training to $2.333^{\circ}$ of abduction post-training. These results indicate that in the population tested, the right side was in significantly more adduction than the left side of the body in both pre and post-testing measures.

\subsection{Vertical Leap, Drop Jump Test}

A Paired Samples T-test indicated a significant difference in time $(\mathrm{p}=0.005)$, pre-test and post-test. The post-test vertical leap had a marginally lower mean than pre-test. The pre-test mean was 9.5766 and the post-test mean was 8.8603 . This results in a $1.8034 \mathrm{~cm}$ difference between pre-test and post-test, indicating that jump height decreased after intervention.

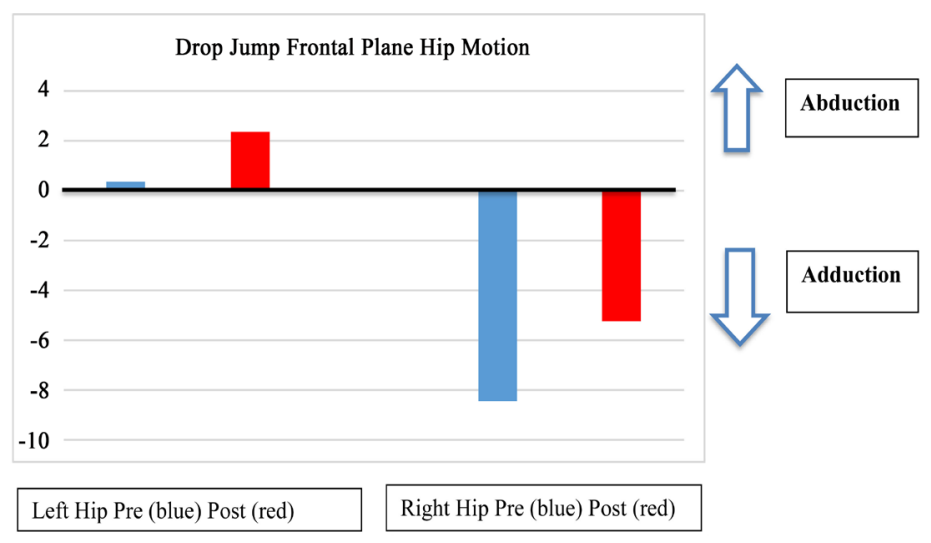

Figure 3. Frontal plane hip motion. Significant differences $(p=0.002)$ were seen in the post training data for both hips. 


\section{Discussion}

Female athletes are at a high risk for ACL injury due to multifactorial considerations which increase ACL strain through the knee complex when the lower extremity is loaded in closed-chained maneuvers, which can result from combinations of small peak flexion moment at the knee and high peak adduction moments at the hip [25]. The present study found significant increases in peak values of knee flexion, hip flexion, and hip abduction respectively during a drop-jump task following a neuromuscular re-education training intervention, suggesting that participants were able to modify their body mechanics to increase sagittal plane protection in the lower quarter. Improved sagittal protection is associated with decreased anterior tibial translation and decreased valgus moments of the knee, and therefore decreased risk of ACL trauma [25]. The direct connection between ACL injury and increased valgus loading has been clearly demonstrated by in vivo and cadaver research [19].

Increased knee flexion accompanied by increased hip flexion is important in facilitating a soft landing technique, which involves increased activation of the gluteus maximus and hamstrings musculature and a decreased contraction of the quadriceps, decreasing knee extensor moments and thereby allowing for more energy to be absorbed and dispersed at the hip joints [22] [23]. This hip-dominant landing technique in combination with hip strategies that minimize valgus loading moments at the knee collectively attenuate strain on passive supporting structures of the knee by correcting high-risk movement behavior commonly exhibited during deceleration and rapid change of direction moments [25], placing less load specifically on the ACL for any given loading action from the ground. The results of the current study demonstrate that these movement behaviors are modifiable and susceptible to positive influence with neuromuscular training intervention in adolescent female soccer players over an 8-week period.

Specifically, the outcomes described with the Drop Jump Test among the subjects after training warrant in depth consideration. The training intervention implemented in the current study focused on both strengthening of the proximal hip stabilizers and neuromuscular re-education techniques of the lower quarter to optimize movement behavior during closed kinetic chain loading tasks. Neuromuscular deficits in strength and coordination of the stabilizing muscles of the knee have been shown to predispose the athlete to injury, and these factors are of even greater importance to address in those who exhibit high-risk movement strategies [9] [16] [23] [24]. The significant increase in peak hip and knee flexion in addition to peak hip abduction (decreased hip adduction) observed with landing in the subjects indicates the successful adoption of the soft-landing strategy and the foundational integration of a hip-strategy technique, maintaining the femur closer to neutral in the frontal plane of motion safely during a peak loading moment. These outcomes are most closely attributed to increased volitional recruitment of neuromuscular stabilizing structures of hip in addition to the hamstrings musculature. These findings indicate a more optimal biomechanical profile in the participants after the training intervention that is strongly 
associated with a decreased risk of ACL injury.

Finally, the results indicated a marginal decrease in jump height of $1.8034 \mathrm{~cm}$ between pre-test and post-test measures. While even the smallest differences in performance related measures in sport warrant consideration, it should be taken into account that subjects were in the process of implementing novel neuromotor techniques at the time of post-test measures and may still be technically categorized in a learning phase of development during the time of testing. With the acquisition of any new technique, there is an associated period in which the new skill must be integrated from initial conceptualization training to functional utilization, during which a transient decrease in speed and power demonstrated is expected as the demand for cognitive resources is temporarily increased to meet the nuances of the new technique. An additional rationale for the decrease in jump height observed is that the training intervention was focused primarily on the deceleration (landing) phase of the jump, and secondarily on the ascending phase. The priority was to improve the biomechanics of the jump landing for the purposes of safety and economy of motion, and performance gains would only be expected after initial mastery is achieved and technique is integrated into regular training thereafter.

One major limitations of the present study was the inability of researchers to control for activities outside of the intervention curriculum that subjects took part in between pre and post-testing assessments. Some of the athletes participated in games or practice on the same day of post-testing procedures, which may have influenced their performance. This was unavoidable given the structural constraints of the research design, yet could have influenced the results, particularly in consideration of the understanding that fatigue is strongly associated with athletic performance and neuromuscular control.

A second limitation includes the group training methodology employed for training purposes, addressing nuances in a domain where one-on-one intervention in a clinical setting is standard practice. Further, it should be acknowledged that the findings of this study are most specifically generalizable to adolescent female soccer players. The subjects tested were all physically active and not injured at the onset of the intervention, and caution is warranted when implementing advanced training methods while rehabilitating from injury. Future studies which investigate the effects of similar interventions in different age groups, in different types of sports, or including males and females stand to contribute further to this research topic. Finally, longitudinal studies with reliable injury tracking systems in place would shed invaluable light on the long-term efficacy of such training interventions and may provide further support for their implementation.

\section{Conclusions}

The results of this study showed that a short duration (six, $90 \mathrm{~min}$, sessions occurring over 8 weeks) training intervention can significantly influence the landing kinematics in adolescent female soccer players during a drop jump assess- 
ment. Increased peak values of hip and knee flexion along with decreased hip adduction during landing indicate a more optimal loading technique associated with decreased risk of ACL injury.

There is a clear need for reliable means to identify athletes who are at a high risk of ACL injury, and to develop training programs which attenuate risk for trauma during athletic competition. In the present study, peak range of motion measures at the hip and knee joints during a drop jump landing task were assessed before and after a six-session neuromuscular training intervention using an inertial system. These measurements were employed to assess the quality of movement and the ability of training to influence the biomechanics of the hip and knee during the landing phase of jumping in adolescent female soccer players. The inertial motion analysis system was a useful screening tool for identifying high-risk biomechanics that must be addressed to develop successful ACL injury prevention programs. Specifically, it appears that high-risk movement behaviors during the deceleration phase of dynamic loading moments associated with ACL injury are identifiable and subject to change with a neuromuscular training intervention. The findings of the current study provide useful information for the utilization of 3D motion analysis technologies in identifying risk for injury and the growth of intervention programs aimed to prevent ACL injury in female athletes. Future research should focus on developing the most effective training exercises to implement specific to various populations in need, and on the long-term effectiveness of such interventions.

\section{Funding Statement}

There were no outside funding sources used with this project. All authors contributed their time for the completion of this project.

\section{Conflicts of Interest}

The authors declare that there is no conflict of interest regarding the publication of this paper.

\section{References}

[1] Prodromos, C.C., Han, Y., Rogowski, J., Joyce, B.S. and Shi, K. (2007) A Meta-Analysis of the Incidence of Anterior Cruciate Ligament Tears as a Function of Gender, Sport, and a Knee Injury-Reduction Regimen. The Journal of Arthroscopic \& Related Surgery, 23, 1320-1325. https://doi.org/10.1016/j.arthro.2007.07.003

[2] Brophy, R.H., Stepan, J.G., Silvers, H.J. and Mandelbaum, B.R. (2015) Defending Puts the Anterior Cruciate Ligament at Risk during Soccer. Sports Health: A Multidisciplinary Approach, 7, 244-249. https://doi.org/10.1177/1941738114535184

[3] Mather, R.C., Koenig, L., Kocher, M.S., et al. (2013) Societal and Economic Impact of Anterior Cruciate Ligament Tears. The Journal of Bone \& Joint Surgery, 95, 1751-1759. https://doi.org/10.2106/JBJS.L.01705

[4] Gelber, A.C., Hochberg, M.C., Mead, L.A., et al. (2000) Joint Injury in Young Adults and Risk for Subsequent Knee and Hip Osteoarthritis. Annals of Internal Medicine, 133, 321-328. 
[5] Oiested, B.E., Engebresten, L. and Storheim, K.R.M. (2009) Knee Osteoarthritis after Anterior Cruciate Ligament Injury. The American Journal of Sports Medicine, 37, 1434-1443. https://doi.org/10.1177/0363546509338827

[6] Lohmander, L.S., Ostenberg, A., Englund, M. and Roos, H. (2004) High Prevalence of Knee Osteoarthritis, Pain, and Functional Limitations in Female Soccer Players Twelve Years after Anterior Cruciate Ligament Injury. Arthritis \& Rheumatism, 50, 3145-3152. https://doi.org/10.1002/art.20589

[7] Brophy, R.H., et al. (2012) Return to Play and Future ACL Injury Risk after ACL Reconstruction in Soccer Athletes from the Multicenter Orthopaedic Outcomes Network (MOON) Group. The American Journal of Sports Medicine, 40, 2517-2522. https://doi.org/10.1177/0363546512459476

[8] McNair, P.J. and Marshall, R.N. (1990) Important Features Associated with Acute Anterior Cruciate Ligament Injury. The New Zealand Medical Journal, 103, 537-539.

[9] Boden, B.P., Dean, G.S., et al. (2000) Mechanisms of Anterior Cruciate Ligament Injury. Orthopedics, 23, 573-578. https://doi.org/10.3928/0147-7447-20000601-15

[10] Fauno, P. and Jakobsen, B.W. (2006) Mechanism of ACL Injuries in Soccer. International Journal of Sports Medicine, 27, 75-79. https://doi.org/10.1055/s-2005-837485

[11] Feagin, J.A.L.K. (1985) Mechanism of Injury and Pathology of Anterior Cruciate Ligament Injuries. Orthopedic Clinics of North America, 16, 41-45.

[12] Shimokochi, Y. and Shultz, S.J. (2008) Mechanisms of Noncontact Anterior Cruciate Ligament Injury. Journal of Athletic Training, 43, 396-408. https://doi.org/10.4085/1062-6050-43.4.396

[13] McLean, S.G. and Samorezov, J. (2009) Fatigue-Induced ACL Injury Risk Stems from a Degradation in Central Control. Medicine \& Science in Sports \& Exercise, 41, 1661-1672. https://doi.org/10.1249/MSS.0b013e31819ca07b

[14] Silvers, H.J. and Mandelbaum, B.R. (2007) Prevention of Anterior Cruciate Ligament Injury in the Female Athlete. British Journal of Sports Medicine, 41, 152-159. https://doi.org/10.1136/bjsm.2007.037200

[15] Arendt, E.A., Agel, J. and Dick, R. (1999) Anterior Cruciate Ligament Injury Patterns Among Collegiate Men and Women. Journal of Athletic Training, 34, 86-92. http://www.ncbi.nlm.nih.gov/pmc/articles/PMC1322895/

[16] Griffin, B.L.Y., Albohm, M.J., Arendt, E.A., et al. (2006) Understanding and Preventing Noncontact Anterior Cruciate Ligament Injuries: A Review of the Hunt Valley II Meeting. The American Journal of Sports Medicine, 34, 1512-1532.

[17] Renstrom, P., Ljungqvist, A., Arendt, E., et al. (2008) Non-Contact ACL Injuries in Female Athletes: An International Olympic Committee Current Concepts Statement. British Journal of Sports Medicine, 42, 394-412. http://bjsm.bmj.com/content/42/6/394.abstract https://doi.org/10.1136/bjsm.2008.048934

[18] Hewett, T.E., Myer, G.D., Ford, K.R., et al. (2005) Biomechanical Measures of Neuromuscular Control and Valgus Loading of the Knee Predict Anterior Cruciate Ligament Injury Risk in Female Athletes: A Prospective Study. The American Journal of Sports Medicine, 33, 492-501. https://doi.org/10.1177/0363546504269591

[19] Hewett, T.E., Ford, K.R., et al. (2006) Anterior Cruciate Ligament Injuries in Female Athletes: Part 2, a Meta-Analysis of Neuromuscular Interventions Aimed at Injury Prevention. The American Journal of Sports Medicine, 34, 490-498. https://doi.org/10.1177/0363546505282619 
[20] Herzog, M.M., Marshall, S.W., Lund, J.L., Pate, V. and Mack, C.D. (2017) Incidence of Anterior Cruciate Ligament Reconstruction among Adolescent Females in the United States, 2002 through 2014. JAMA Pediatrics, 171, 808-810.

[21] Loyola University Health System (2014) Young Female Athletes Suffering Epidemic of ACL Knee Injuries. Science Daily. https://www.sciencedaily.com/releases/2014/04/140430091418.htm

[22] Pollard, C.D., Sigward, S.M. and Powers, C. (2010) Limited Hip and Knee Flexion during Landing Is Associated with Increased Frontal Plane Knee Motion and Moments. Clinical Biomechanics, 25, 142-146. https://doi.org/10.1016/j.clinbiomech.2009.10.005

[23] Malinzak, R.A., Colby, S.M., Kirkendall, D.T., Yu, B. and Garrett, W.E. (2001) A Comparison of Knee Joint Motion Patterns between Men and Women in Selected Athletic Tasks. Clinical Biomechanics, 16, 438-445. https://doi.org/10.1016/S0268-0033(01)00019-5

[24] Ford, K.R., Myer, G.D. and Hewett, T.E. (2003) Valgus Knee Motion during Landing in High School Female and Male Basketball Players. Medicine \& Science in Sports \& Exercise, 35, 1745-1750. https://doi.org/10.1249/01.MSS.0000089346.85744.D9

[25] Sigward, S.M. and Powers, C.M. (2006) The Influence of Gender on Knee Kinematics, Kinetics and Muscle Activation Patterns during Side-Step Cutting. Clinical Biomechanics, 21, 41-48. https://doi.org/10.1016/j.clinbiomech.2005.08.001

[26] Wolman, R. (2009) Epidemiology: Knee Injuries in Female Athletes. Nature Reviews Rheumatology, 5, 527-528. https://doi.org/10.1038/nrrheum.2009.194

[27] Hewett, T.E., Myer, G.D. and Ford, K. (2006) Anterior Cruciate Ligament Injuries in Female Athletes: Part 1, Mechanisms and Risk Factors. The American Journal of Sports Medicine, 34, 299-311. https://doi.org/10.1177/0363546505284183

[28] Ireland, M.L. (2002) The Female ACL: Why Is It More Prone to Injury? Orthopedic Clinics of North America, 33, 637-651. https://doi.org/10.1016/S0030-5898(02)00028-7

[29] Noraxon, U. Noraxon. http://www.noraxon.com/products/3d-motion-capture/ 